\title{
Speech perception in preschoolers at family risk for dyslexia: Relations with low-level auditory processing and phonological ability
}

\author{
Bart Boets $^{\mathrm{a}, *}$, Pol Ghesquière ${ }^{\mathrm{a}}$, Astrid van Wieringen ${ }^{\mathrm{b}}$, Jan Wouters ${ }^{\mathrm{b}}$ \\ ${ }^{a}$ Centre for Disability, Special Needs Education and Child Care, University of Leuven, Vesaliusstraat 2, B-3000 Leuven, Belgium \\ ${ }^{\mathrm{b}}$ Laboratory for Experimental ORL, Department of Neurosciences, University of Leuven, Belgium
}

Accepted 24 June 2006

Available online 2 August 2006

\begin{abstract}
We tested categorical perception and speech-in-noise perception in a group of five-year-old preschool children genetically at risk for dyslexia, compared to a group of well-matched control children and a group of adults. Both groups of children differed significantly from the adults on all speech measures. Comparing both child groups, the risk group presented a slight but significant deficit in speech-in-noise perception, particularly in the most difficult listening condition. For categorical perception a marginally significant deficit was observed on the discrimination task but not on the identification task. Speech parameters were significantly related to phonological awareness and low-level auditory measures. Results are discussed within the framework of a causal model where low-level auditory problems are hypothesized to result in subtle speech perception problems that might interfere with the development of phonology and reading and spelling ability.
\end{abstract}

(C) 2006 Elsevier Inc. All rights reserved.

Keywords: Speech-in-noise perception; Categorical perception; Auditory processing; Phonology; Dyslexia; Preschoolers

\section{Introduction}

Every beginning reader of an alphabetic writing system is confronted with the challenge to learn to segment the quasi-continuous speech signal in distinct and specified phoneme units in order to map them upon their corresponding graphemes. Whereas the majority of readers fairly easily establishes stable grapheme to phoneme relations, a small group of about $5-10 \%$ of children and adults is not able to master fluent literacy skills in spite of normal intelligence, adequate environment, and educational opportunities. At present, it is well established that these reading and spelling impairments, characteristic of dyslexia, are related to specific deficits in the representation and use of phonological information (for a review see Rack, 1994; Snowling, 2000). Phonological deficits have been demonstrated in

\footnotetext{
${ }^{*}$ Corresponding author. Fax: +32 0 16/32 5933.

E-mail address: bart.boets@ped.kuleuven.be (B. Boets).
}

three broad areas: phonological awareness (e.g. Manis, Custodio, \& Szeszulski, 1993), retrieval of phonological codes from long-term memory (e.g. Bowers \& Swanson, 1991; Wolf, 1986), and verbal short-term memory (e.g. Catts, 1989).

In recent years, it has also been suggested that subjects with dyslexia show problems in speech perception (for a review see McBride-Chang, 1995). They tend to have difficulty extracting discrete phonological representations from phonetic features embedded within the speech signal (Manis et al., 1997; Serniclaes, Sprenger-Charolles, Carré, \& Demonet, 2001). Additionally, there is also some evidence for a more fundamental deficit in low-level auditory information processing. In particular, it has been suggested that dyslexics have problems processing short, rapidly presented and dynamic changing acoustic stimuli (e.g. Farmer \& Klein, 1995; McArthur \& Bishop, 2001; Menell, McAnally, \& Stein, 1999; Talcott et al., 2002, 1999, 2000; Tallal, 1980; Van Ingelghem et al., 2005; Witton et al., 1998). One hypothesis maintains that this fundamental deficit in 
perceiving auditory temporal cues might be at the basis of the speech perception problem. Consequently, it has been hypothesized that the resulting speech perception problem might interfere with the development of fine-grained phonological representations, which in turn impacts upon reading and spelling development (Talcott \& Witton, 2002; Tallal, 1980, 1984; Watson \& Miller, 1993; Wright et al., 1997).

The postulation of this causal 'auditory temporal processing deficit' hypothesis did not only provoke an intense, yet ongoing scientific debate (e.g. Blomert \& Mitterer, 2004; Denenberg, 1999; Mody, Studdert-Kennedy, \& Brady, 1997; Nittrouer, 1999; Rosen \& Manganari, 2001), but also induced an exponential increase of studies. However, in spite of the developmental aspect implied in the hypothesis, most of these studies were carried out on adult subjects, a minority on school-aged children and only very few on preschool children (e.g. Lyytinen et al., 2001). In this paper, we report data from a longitudinal study that explores the development of basic auditory skills, speech perception, phonological ability and reading skill in a group of fiveyear-old preschool children genetically at risk for dyslexia, compared to a group of well-matched control children. In a previous paper (Boets, Wouters, van Wieringen, \& Ghesquière, 2006), we already reported that the children at family risk presented a significant deficit in phonological awareness and letter knowledge, but not in rapid automatic naming nor verbal short-term memory. They were also unimpaired on auditory measures for gap-in-noise detection, $2 \mathrm{~Hz} F M$ detection and tone-in-noise detection, although thresholds for tone-in-noise and FM detection were significantly related to phonological awareness. In the current paper, we will focus upon speech perception abilities, assessed in the same group of preschool children. In particular, we consider the question whether a deficit in speech perception may already be observable in preschool children at risk for dyslexia and we investigate the relationship between speech perception and auditory and phonological processing.

Dyslexia research has mainly used two major experimental paradigms to study speech perception: (i) the perception of speech presented in background noise and (ii) categorical perception of stop consonants.

Although it is widely acknowledged that subjects with reading problems have difficulty with the perception of speech under noisy listening conditions, so far only a limited number of empirical studies have addressed this issue in well-defined clinical groups. Brady, Shankweiler, and Mann (1983) showed that nine-year-old poor readers had more difficulty in repeating words presented in noise than age-matched controls, though both groups did not differ in the perception of speech without noise. Similarly, Chermak, Vonhof, and Bendel (1989) found that adults with broadly defined learning disabilities had poorer word identification in noise than a control group of adults without learning disabilities. The same results were obtained by Watson and Miller (1993), presenting CV syllables in a background of cafeteria noise. Two studies presenting meaningful sentences in noise found that children with hearing or language impairments (Stollman, Kapteyn, \& Sleeswijk, 1994) or with language-based learning disabilities (Bradlow, Kraus, \& Hayes, 2003) showed poorer overall sentence-innoise perception than a non-affected control group. Moreover, both studies demonstrated that children with language problems were more affected by more adverse listening conditions than the control group.

Recently, some neurophysiologic studies also provided evidence for anomalies in dyslexics' neural encoding of speech stimuli embedded in background noise. Wible, Nicol, and Kraus (2002) demonstrated a deficiency in the neural representation of repeated speech stimuli in noise but not in quiet in 11-year-old children diagnosed with reading problems. Moreover, the robustness of the physiologic response in noise was significantly correlated with behavioral measures of speech discrimination, with a phonological composite measure and with spelling ability. In the same way, Cunningham, Nicol, Zecker, Bradlow, and Kraus (2001) reported that a group of reading disabled children (aged 10-13 years) showed poorer speech discrimination coupled with diminished neurophysiologic cortical and subcortical responses in background noise compared to normal children. There were no differences between the groups in quiet listening conditions, neither in behavioral perception, nor in electrophysiology. Similar results, indicative of abnormal timing of cortical responses to speech stimuli in background noise in learning-impaired children, were reported by Warrier and colleagues (Warrier, Johnson, Hayes, Nicol, \& Kraus, 2004).

Taken together, even though these studies are diffuse in terms of methodology and participant inclusion criteria, the findings indicate that subjects with dyslexia or more broadly defined learning or language impairments have problems perceiving speech in the presence of background noise. However, evidence of a speech-in-noise perception deficit is not yet unequivocal since some studies failed to find a discrepancy (Pennington, Van Orden, Smith, Green, \& Haith, 1990; Snowling, Goulandris, Bowlby, \& Howell, 1986 ) or could only demonstrate it under very specific noisy listening conditions (viz. intermittent noise, see Bailey, Griffiths, Hill, Brent, \& Snowling, 2002).

The categorical perception experiments are based on the general principle that perceptual discrimination between speech sounds belonging to different phoneme categories is better than that between sounds falling within the same category, irrespective of the magnitude of the acoustic (physical) difference between the sounds (Liberman, Harris, Hoffman, \& Griffith, 1957). In identification experiments this is expressed in the discontinuous perception of a series of speech stimuli that vary continuously along a single acoustic dimension. Normal listeners exhibit a strong tendency towards categorical perception of speech along certain phonetic dimensions, such as place of articulation (e.g. /ba/ vs. /da/) or voicing status (e.g. /ba/ vs. /pa/). Several studies suggest that children and adults with dyslexia are 
less categorical than average readers in the way they perceive phonetic contrasts (Breier, Fletcher, Denton, \& Gray, 2004; Godfrey, Syrdal-Lasky, Millay, \& Knox, 1981; Lieberman, Meskill, Chatillon, \& Schupack, 1985; Maassen, Groenen, Crul, Assman-Hulsmans, \& Gabreëls, 2001; Manis et al., 1997; Reed, 1989; Serniclaes et al., 2001, Serniclaes, Van Heghe, Mousty, Carré, \& Sprenger-Charolles, 2004; Steffens, Eilers, Gross-Glenn, \& Jallad, 1992; van Beinum, Schwippert, Been, van Leeuwen, \& Kuijpers, 2005; Werker \& Tees, 1987). Some studies could even demonstrate a weaker categorical perception in toddlers (Gerrits, 2003) and six-month-old infants (Richardson, Leppänen, Leiwo, \& Lyytinen, 2003) at family risk for dyslexia. However, a minority of studies obtained non-significant results (Blomert \& Mitterer, 2004; Brandt \& Rosen, 1980; Nittrouer, 1999) or only observed the categorical perception deficit in the most severely phonologically or linguistically impaired dyslexics (Joanisse, Manis, Keating, \& Seidenberg, 2000; Manis et al., 1997).

Notwithstanding the empirical evidence that speech perception measures are able to differentiate relatively reliably between adult and school-aged dyslexic and normal reading subjects, the differentiating and predictive power of these tasks has been studied only limitedly in preschoolers. In this study we want to deal with this issue by examining categorical perception and speech-in-noise perception in two contrasting groups of preschool children. Further, by correlating the speech data with previously collected auditory and phonological data, we aim to shed light on the relation between low-level auditory processing, speech perception and phonological ability. A specific advantage of studying preschool children, who never received any formal reading instruction, is that it allows ruling out the possibility that group differences or interrelations would merely be a result of differences in literacy skill or reading experience.

\section{Method}

\subsection{Participants}

Sixty-two five-year-old children attending the last year of kindergarten ${ }^{1}$ were included in the study (36 boys/26 girls). Half of the participants were children of 'dyslexic families', the so-called high-risk group (HR); the other half were control children of 'normal reading families', the socalled low-risk group (LR). Since dyslexia tends to run strongly in families, preschoolers with dyslexic relatives are more likely to develop reading problems. Gilger, Pennington, and DeFries (1991) estimate that roughly between $30 \%$ and $50 \%$ of such children will become reading disabled. Moreover, in view of the fact that the HR group already demonstrated a significant deficit in phonological awareness and letter knowledge (Boets et al., 2006), and since

\footnotetext{
${ }^{1}$ In the Belgian school system, formal instruction starts in grade 1 at six years. This means in kindergarten no reading instruction is offered.
}

these measures have consistently been proven to be the best single preschool predictors of literacy development (Scarborough, 1998), it is very likely that this group will contain a disproportional high number of future cases of dyslexia.

All children were native Dutch speakers without any history of brain damage, long-term hearing loss or visual problems. Additionally, at the moment of data collection they did not present any gross hearing deficiencies (audiometric pure-tone average $<25 \mathrm{~dB} \mathrm{HL}$ ). The HR children were selected on a basis of having at least one first-degree relative with a diagnosis of dyslexia. The LR children showed no history of speech or language problems and none of their family members suffered any learning or language problem. For every individual HR child the best matching LR control child was selected based on five criteria: (1) educational environment, i.e. same nursery school, (2) gender, (3) age, (4) nonverbal intelligence, and (5) parental educational level. Nonverbal intelligence was assessed by an adapted version of the Raven Coloured Progressive Matrices (RCPM) (Raven, Court, \& Raven, 1984), a collective nonverbal intelligence test measuring spatial reasoning. Parental educational level was assessed using the ISCEDscale (International Standard Classification of Education by UNESCO, 1997), by converting classifications on the original seven-point scale to a three-point scale. At the time of collecting the speech data, the mean age for both the HR and LR group was 5 years and 8 months, not being statistically different [paired $t(30)=0.22, p=.83$ ]. The nonverbal IQ scores were slightly above population average (107 for HR group and 111 for LR group) and did not differ significantly [paired $t(30)=1.88, p=.07]$. Fisher's exact test also confirms that both groups did not differ in frequency distribution of the different educational categories $(p=.71$ for maternal and $p=.43$ for paternal educational level). Further details about the participants and the selection procedure are described in Boets et al. (2006).

For reasons of comparison, the speech tests were also administered to a group of eleven normal hearing and reading adults, aged $24-43$ years ( 5 males $/ 6$ females).

\subsection{Tasks and materials}

\subsubsection{Tests for speech perception}

2.2.1.1. Speech-in-noise perception task. In the speech-innoise perception task, seven lists of 10 high-frequency monosyllabic words (taken from the Göttingerlist II, recorded by Wouters, Damman, \& Bosman, 1994; see also van Wieringen $\&$ Wouters, 2005) were presented monaurally with an interstimulus interval of $7 \mathrm{~s}$. Simultaneously, a continuous stationary speech noise with an identical spectrum as the average spectrum of the word lists was presented to the same ear, at a fixed level of $70 \mathrm{~dB}$ SPL. Words were presented at $-1,-4$ and $-7 \mathrm{~dB}$ signal-to-noise ratio (SNR). Before administration of the six test lists $(3 \times 2)$, one list was presented at an SNR of $+4 \mathrm{~dB}$ as a practice list. The child's task was to repeat the words as accurately as possible, resulting in a percentage correct word score for every test list. 
2.2.1.2. Categorical perception. A ten-point speech continuum ranging from $/ \mathrm{bAk} /$ to $/ \mathrm{dAk} /$ (i.e. the Dutch word for 'box/basket' and 'roof') was presented by means of a categorical perception task. For every child, a 2AFC forcedchoice identification task was administered, followed by a same-different (AX) discrimination task. Stimuli were identical to the ones developed and described by van Beinum et al. (2005). Stimuli were based on natural speech and were constructed by linearly interpolating the transition of the second formant $\left(F_{2}\right)$ from $/ \mathrm{b} /$ to $/ \mathrm{d} /$. The manipulated part of the signal was a $100 \mathrm{~ms}$ interval at the beginning of the vowel. There were ten interpolation steps and the $F_{2}$ onset ranged from 1100 to $1800 \mathrm{~Hz}$. The /bAk/-starting point was characterized by a constant $F_{2}$ value of $1100 \mathrm{~Hz}$. For each intermediate signal, the $F_{2}$ onset was gradually situated at higher frequencies, making the fall of the transition steeper in every step. At the /d/-endpoint of the continuum the transition was falling from 1800 to $1100 \mathrm{~Hz}$. Stimuli were presented monaurally at a comfortable listening level of $70 \mathrm{~dB}$ SPL.

In the identification task, subjects were sitting in front of a computer screen, depicting a picture of a basket and a roof, representing the possible response options (/bAk/ vs. / $\mathrm{dAk} /$ ). The 10 stimuli of the continuum were presented twelve times in a random order, the same stimulus not being presented more than two consecutive times. The child was instructed to repeat the perceived word and point to the corresponding picture. Participants were given an unlimited time to respond. No direct feedback was given. The task was preceded by a pre-test, presenting each of the two endpoint stimuli five times.

In the discrimination task, subjects were instructed to listen to two stimuli presented with an ISI of $600 \mathrm{~ms}$ and to determine whether they sounded 'the same' or 'different'. Children had to respond by pointing to one of two pictures on the screen representing "the same" or "different". No direct feedback was given. In order to obtain a bias-free measure of discriminability, the task contained physically different as well as identical pairs. The different pairs, comprising each of the seven 3-step comparisons (1-4, 2-5, 3-6, etc), were presented 6 times; the identical pairs $(1-1,2-2$, etc.) were presented twice. The pairs of stimuli were presented in two blocks of 31 stimuli $(3 \times 7+1 \times 10)$. Presentation order of the stimuli was randomized and the internal order of a different stimulus pair was balanced over both series. The task was preceded by ten practice items, comprising pairs that were clearly different or identical.

Stimulus presentation and response registration was controlled by E-prime, a software module for psychological experiments (Schneider, Eschman, \& Zuccolotto, 2002). In order to make the rather boring psychophysical tests more interesting and child friendly, they were integrated in an interactive videogame with an introductory and concluding movie and animated buttons.

For both the categorical perception task and the speechin-noise perception task, as well as for the other auditory psychophysical tasks, stimuli were presented monaurally over calibrated TDH-39 headphones using the integrated audio PC-card from a portable computer routed to an audiometer (Madsen OB622).

\subsubsection{Phonological tests}

Phonological skills were assessed by a test battery comprising eight tests (see Boets et al., 2006). A principal component factor analysis with varimax rotation confirmed that the battery reflected the three traditional phonological domains (Wagner \& Torgesen, 1987): (a) phonological awareness: high loadings from first-sound and end-sound identity task, rhyme identity task and simple rhyme task, (b) rapid automatic naming: high loadings from both the colors and objects rapid automatic naming tasks and (c) verbal short-term memory: high loadings from the nonword repetition task and the digit span forward task. Details about the composition of the battery can be found in Boets et al. (2006); in this paper we will only refer to the factor scores.

\subsubsection{Tests for low-level auditory processing}

Auditory processing was assessed by means of three psychophysical threshold tests (see Boets et al., 2006). With the gap-in-noise detection task (GAP), we tested the hypothesis of a deficit in 'rapid and brief' temporal processing. With the $2 \mathrm{~Hz}$ frequency modulation detection task (FM), we verified the hypothesis of a deficit in the processing of 'dynamic stimuli'. With the tone-in-noise detection task $(T N)$, a non-temporal control task, we verified the temporal specificity of any observed auditory deficit.

In the GAP-detection test, subjects had to detect a silent interval (gap) in a white noise stimulus. Threshold was defined as the minimum gap length required for detecting the silent interval. In the FM-detection test participants had to detect a $2 \mathrm{~Hz}$ sinusoidal frequency modulation of a $1 \mathrm{kHz}$ carrier tone with varying modulation depth. Threshold was defined as the minimum depth of frequency deviation required to detect the modulation. In the TN-detection task participants had to detect two pure tone pulses $(1 \mathrm{kHz}$, length $=440 \mathrm{~ms}$ ) within a one-octave noise signal, centered around $1 \mathrm{kHz}$ (from 707 to $1414 \mathrm{~Hz}$, length $=1620 \mathrm{~ms}$ ). Threshold was defined as the lowest signal-to-noise ratio (SNR) required for detecting the tone pulses. For all three psychophysical tests, thresholds were estimated using a three-alternative forced-choice oddity paradigm embedded within an interactive computer game with animation movies (Laneau, Boets, Moonen, van Wieringen, \& Wouters, 2005). The length of the gap, the depth of modulation and the amplitude of the sinusoidal pulses were adjusted adaptively using a two-down, one-up rule, which targeted the threshold corresponding to $70.7 \%$ correct responses. For each participant three threshold estimates were determined for every experiment. For the correlation data we present here, the average of the best and second best threshold was used as an indicator of auditory sensitivity. A detailed description of the stimuli, procedure and results can be found in Boets et al. (2006). 
Data collection was carried out by qualified psychologists and audiologists. Testing took place in a quiet room at the children's school. Since the LR child was selected from the HR child's classmates, both children could always be tested in exactly the same circumstances.

\subsection{Statistical analysis}

First, both groups of children were compared to each other. To account for the clustered nature of the data (i.e. matched pairs), data were analyzed in a pair wise manner using Mixed Model Analysis (MMA) (Littell, Stroup, \& Freund, 2002). ${ }^{2}$ Although both groups did not show a significant difference on any of the matching criteria, any possible influence of age, nonverbal intelligence or parental educational level was ruled out by additionally controlling for these variables. As such, a series of (repeated) MMA's was calculated with pair number as a random variable (1-31) and participant group (HR vs. LR) as the fixed between-subject variable. Age, nonverbal IQ and parental educational level were added as fixed (co)variables. Second, child and adult data were compared to each other using MMA as well. For this comparison no random factor or covariables were specified. Post-hoc analyses were corrected for multiple comparisons using Tukey procedure. Third, to extract the essential parameters, the speech-innoise data and the identification data were submitted to a logistic fitting and groups were compared on the resulting parameters. Prior to analysis, the slope-parameters of these curves were $\log _{10}$-transformed to obtain normally distributed data. In order to take into account the variable quality of the fits, the inverse standard error of the estimated parameters was added to the model as a weight variable. Finally, in order to determine the relations between speech perception on the one hand and auditory and phonological processing on the other, Pearson correlations were calculated, partialed out for individual differences in nonverbal intelligence and with the inverse standard error of the estimated parameters added as a weight variable.

\section{Results}

\subsection{Speech-in-noise perception}

Prior to analysis, data from one subject were removed because of irregularities during testing. Average results of the speech-in-noise perception test are depicted in Fig. 1. A paired wise Repeated Measures MMA with proportion correctly perceived words as dependent variable, group as

\footnotetext{
${ }^{2}$ Traditional statistics (e.g. ANOVA and regression analysis) require independence of errors, but this assumption is not met in a dependent sample design. Mixed models allow modelling this dependent nature of the data. By entering pair number as a random factor in all analyses, these models are an extension of the paired $t$-test, while offering the possibility to include more sophisticated options such as the incorporation of covariates and repeated measurement analyses.
}

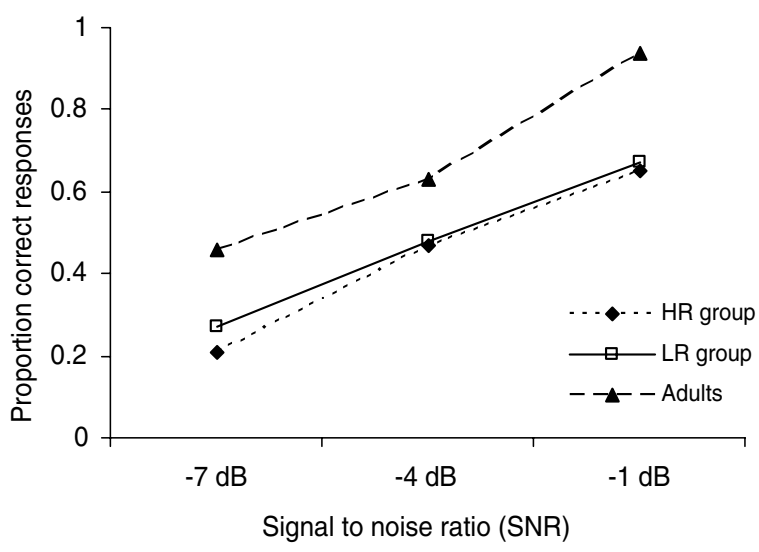

Fig. 1. Mean scores relating the proportion correctly perceived words to the relative level of the presented words (SNR) (HR group: $n=30$, LR group: $n=31$; adult group: $n=8$ ).

between-subject variable (HR vs. LR), SNR as within-subject variable $(-1,-4$ and $-7 \mathrm{~dB}$ SNR) and with the same covariates as mentioned above, revealed a significant effect for group $(p=.04)$, a significant effect for SNR $(p<.0001)$ and no significant group $\times$ SNR interaction effect $(p=.22)$. Post-hoc analysis revealed that both groups only differed at the $-7 \mathrm{~dB}$ SNR-level $(p=.01)$. A Repeated Measures MMA comparing the child data with an adult group $(n=8)$, indicated that both the HR and the LR group differed significantly from the adult group $(p<.0001)$ with the group $\times$ SNR interaction being significant as well $(p=.03)$.

Additionally, to estimate the Speech Reception Threshold (SRT) and the slope of the psychometric function, for every subject a logistic function relating percentage correct responses to SNR level was fitted to the data. The SRT indicates the signal-to-noise ratio required for $50 \%$ correct responses and the slope reflects the rate at which performance changes with changes in the level of the signal. Table 1 shows weighted group means for HR, LR and adult group. In order to take into account the variable quality of the fits, the inverse standard error of the estimated parameters was added to the model as a weight variable. A paired wise weighted MMA with the same covariates as mentioned above demonstrated that both child groups differed significantly for SRT $(p=.04)$ and (log-transformed) slope $(p=.02)$. This means that the HR group required a significantly easier signal-to-noise ratio than the LR group in

Table 1

Descriptive statistics for speech perception tests

\begin{tabular}{lccc}
\hline & HR group & LR group & Adult group \\
\hline Speech-in-noise & & & \\
$N$ & 30 & 31 & 8 \\
Weighted SRT (dB) & $-3.3(1.8)$ & $-3.8(2.2)$ & $-6.4(1.0)$ \\
Weighted slope (\%/dB) & $8.9(2.5)$ & $6.5(1.9)$ & $9.0(1.1)$ \\
Identification & & & \\
$N$ & 25 & 23 & 11 \\
Weighted PSE & $4.2(4.0)$ & $4.4(4.1)$ & $5.9(5.4)$ \\
Weighted slope & $16.9(5.7)$ & $21.3(5.3)$ & $75.5(8.0)$ \\
\hline
\end{tabular}

Standard deviations are given in parentheses after the mean. 
order to perceive $50 \%$ of the presented words correctly. Moreover, the steeper slope in the HR group also indicates a faster deterioration of performance while progressing from easy towards difficult listening conditions. A weighted MMA showed that the adult group differed significantly from both child groups regarding SRT $(p<.0001)$, but for slope only the adult-LR difference was significant (adults vs. LR: $p=.04$; adults vs. HR: $p=.77$ ).

\subsection{Categorical perception-identification}

Only subjects obtaining $70 \%$ correct responses or more on the pre-test were included in the analysis, excluding four subjects $(1 \mathrm{HR} / 3 \mathrm{LR})$. For the remaining 58 subjects, identification curves were visually inspected to evaluate whether they showed a gradual increase in /dAk/-responses while progressing from stimulus 1 to 10 . As a result, two additional subjects (1HR/1LR) were excluded, since they consequently perceived all test stimuli as $/ \mathrm{dAk} /$. For the remaining 56 subjects (see Fig. 2), a paired wise Repeated Measures MMA with 'proportion /dAk/ responses' as dependent variable, group as between-subject variable (HR vs. LR), stimulus as within-subject variable (1-10) and with the same covariates as mentioned above, revealed no significant effect for group $(p=.52)$, a significant effect for stimulus $(p<.0001)$ and no significant group $\times$ stimulus interaction effect $(p=.59)$. Post-hoc analysis revealed that for every single stimulus the group difference was insignificant. In contrast, while comparing the child data with an adult group $(n=11)$, a Repeated Measures MMA indicated that both the HR and the LR group differed significantly from the adult group $(p<.01)$ with the group $\times$ stimulus interaction being significant as well $(p<.01)$.

Furthermore, each individual identification curve was submitted to a logistic fitting relating percentage $/ \mathrm{dAk} /-$ responses to stimulus level, in order to estimate the $50 \%$ cross-over point $(\mathrm{PSE}=$ Point of Subjective Equivalence) and the slope in this point. The PSE indicates the phoneme boundary and the slope indicates the range of uncertainty in distinguishing one phoneme category from another. A

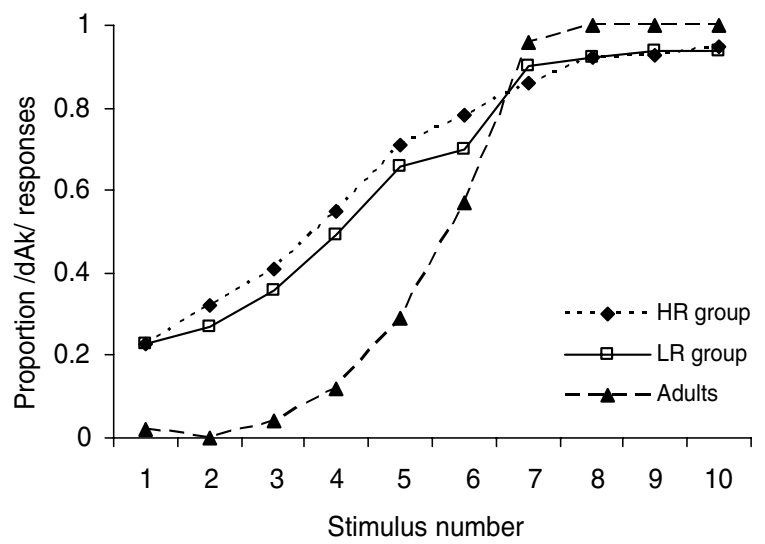

Fig. 2. Mean identification functions relating the proportion /dAk/ responses to the stimulus number of the place-of-articulation continuum (HR group: $n=29$, LR group: $n=27$; adult group: $n=11$ ). high slope value indicates a small uncertainty range and suggests a highly consistent ability to categorise a speech contrast; whereas, a low slope value indicates a large range of uncertainty and suggests difficulties in identifying the seech stimuli (Maassen et al., 2001).

Psychometric functions could be fitted for all 56 participants. However, for eight subjects (4HR/4LR) the phoneme boundary was estimated to be below stimulus 1 , since they even perceived the $/ \mathrm{bAk} /$ endpoint as $/ \mathrm{dAk} /$ in more than $50 \%$ of the presentations. These subjects were excluded from further analysis. For the remaining 48 subjects a paired wise MMA with the same covariates as mentioned above and with the inversed standard error of the estimated parameters as a weight variable showed no significant differences between both groups for PSE $(p=.89)$ and (logtransformed) slope $(p=.21)$. In contrast, in a weighted MMA the adult group differed significantly from both child groups regarding PSE $(p<.001)$, and (log-transformed) slope $(p<.0001)$, indicating that the adult phoneme boundary had moved up towards $/ \mathrm{dAk} /$ and showed a more abrupt perceptual transition (see Table 1 and Fig. 2).

\subsection{Categorical perception-discrimination}

Again, only subjects achieving $70 \%$ correct responses or more on the pre-test were included in the analysis. This resulted in the removal of data of 14 subjects (6HR/8LR). Most of these subjects were also excluded from the identification analysis because they revealed too many errors in that pre-test too or because they did not pass the PSE $>1$ criterion.

In Fig. 3 the mean "discriminability' functions for the HR, LR and adult group are displayed. For every stimulus pair a distribution free and response-bias free index of discriminability ( - ln eta) was calculated according to signal detection theory (Wood, 1976). A paired wise Repeated Measures MMA with 'discriminability' as dependent variable, group as between-subject variable (HR vs. LR), stimulus pair as within-subject variable (1-4, 2-5, etc.) and with the same covariates as mentioned above, showed a marginally

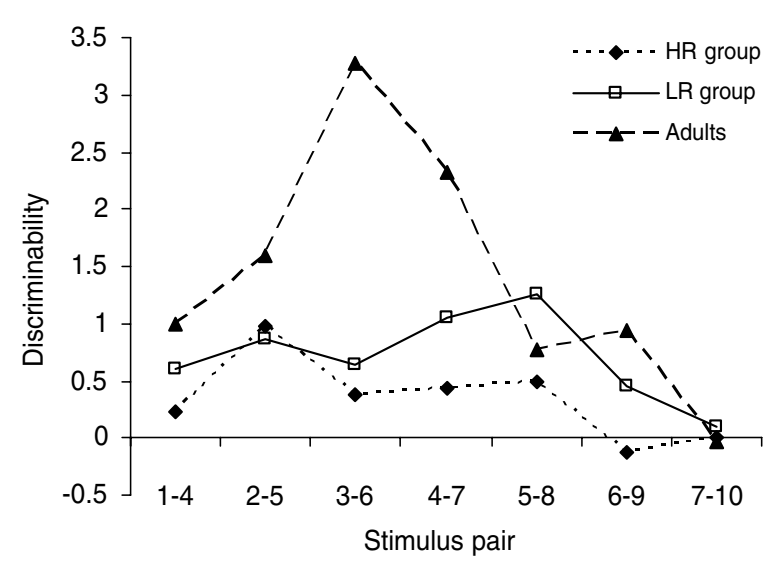

Fig. 3. Mean discrimination scores ( $-\ln$ eta) as a function of stimulus pair (HR group: $n=25$, LR group: $n=23$; adult group: $n=11$ ). 
Table 2

Relations between speech perception and phonological and auditory processing: weighted Pearson correlation coefficients partialed out for individual differences in nonverbal IQ

\begin{tabular}{lcc}
\hline & $\begin{array}{c}\text { Speech reception } \\
\text { threshold }(n=60)\end{array}$ & $\begin{array}{l}\text { Slope phoneme } \\
\text { boundary }^{\mathrm{a}}(n=47)\end{array}$ \\
\hline GAP detection $^{\mathrm{a}}$ & 0.07 & -0.03 \\
FM detection $^{\mathrm{a}}$ & $0.27^{*}$ & -0.22 \\
TN detection & $0.35^{* *}$ & $-0.30^{*}$ \\
Phonological awareness $_{\text {Rapid automatic naming }}$ & $-0.44^{* * *}$ & $0.53^{* * *}$ \\
Verbal short-term memory $^{*}$ & -0.08 & -0.05 \\
\hline
\end{tabular}

Note. ${ }^{\text {a }}$ Scores were $\log _{10}$-transformed prior to analysis.

${ }^{*} p<.05,{ }^{* *} p<.01,{ }^{* * *} p<.001$.

significant effect for group ( $p=.056)$, a significant effect for stimulus pair $(p=.0004)$ and no significant group $\times$ stimulus pair interaction effect $(p=.48)$. Post-hoc analysis revealed that the group difference was significant for pairs 4-7, 5-8 and 6-9. Without adding any covariates, the global repeated group difference also turned out to be significant $(p=.03)$. Comparison of the child data with the adult group $(n=11)$ showed that the HR and LR group differed significantly from the adult group $(p<.001$ and $p<.01$ respectively) with the interaction group $\times$ stimulus pair being significant as well $(p<.0001)$.

\subsection{Relations between speech perception and phonological and auditory processing}

In Table 2 correlations are presented between speech perception ability on the one hand and auditory and phonological processing on the other. Since SRT and the slope of the phoneme boundary are the most informative speech parameters, only correlations with these parameters are presented. Correlations were partialed out for individual differences in nonverbal intelligence and the inversed standard error of the estimated speech parameter was added as a weight variable. Visual inspection of the scatterplots confirmed that the observed relations were proper; i.e. they were not determined by outliers, neither inflated because of aggregating data over extreme groups.

The results of the speech-in-noise test (SRT) were significantly related to phonological awareness, implying that a better speech-in-noise perception corresponded with better phonological processing. Similarly, for categorical perception the abruptness of the phoneme boundary (expressed by the slope parameter) was significantly related to phonological awareness. This implies that a higher slope value, indicative of a small uncertainty range in distinguishing one phoneme category from the other, was related to better phonological awareness. ${ }^{3}$ Considering the relations

\footnotetext{
${ }^{3}$ Noteworthy, another indicator for a relation between categorical perception and phonological awareness is the observation that almost all excluded subjects (based on the $>70 \%$ on the pre-test or PSE $>1$ criterion) score bad on the phonological awareness factor (the average score of all excluded subjects was $.89 S D$ 's below the mean of the LR group).
}

between low-level auditory processing and speech perception, the positive relation between tone-in-noise detection and speech-in-noise perception (SRT) was the most straightforward. Further, sensitivity to FM was also related to speech-in-noise perception, and tone-in-noise detection was related to the abruptness of the perceptual shifting from one phoneme category to the other.

\subsection{Individual deviance analysis}

As mentioned by Manis and colleagues (1997), there are two possible explanations for the mixed pattern of speechperception results observed in literature. First, the group differences between normal reading and reading-impaired subjects are real, but small and therefore difficult to detect. Second, group comparisons might mask larger speech-perception deficits that are found only in specific subgroups of dyslexics. To verify this second option, we carried out analyses at the subject level. To decide which individual did and did not show abnormal performance, we adopted the twostep criterion as suggested by Ramus et al. (2003). Applying this procedure, the criterion for deviance was placed at 1.65 standard deviations of the 'purified' mean of the LR-group (one-sided in the direction indicative of deficiency), after first having excluded all deviant LR-subjects (by applying a similar $1.65 S D$ criterion, usually resulting in the removal of one or two deviant LR subjects). A distribution analysis on the speech results of the 'restricted' LR-group confirmed the normality of the data. Hence, the $1.65 S D$ deviance criterion corresponds to the fifth percentile. A deviance analysis on SRT revealed that 4 out of $30 \mathrm{HR}$ subjects (13\%) showed abnormal performance, whereas in the LR group only 1 out of 31 subjects $(3 \%)$ had abnormal performance. Considering the consistency in categorization of the speech continuum, 11 out of 25 HR subjects (44\%) versus 6 out of 23 LR subjects $(26 \%)$ showed abnormal performance. Although for both speech parameters the HR group presented a higher proportion of deviant subjects, the difference was not significant (Fisher exact test, $p>.05$ ).

Individual $Z$-scores for speech perception parameters and for phonological and auditory measures, relative to the mean of the 'restricted' LR group are depicted in Appendix. Inspection of the individual scores reveals that there is no straightforward regularity or tendency between these measures. For the speech perception data for instance, deficits in categorical perception and speech-in-noise perception appear to be largely mutually unrelated. The same applies for deficits in phonology and low-level auditory processing: some subjects differ only on one of the tasks, others on two or three, but without consistency (see also Boets et al., 2006). To investigate the relation between speech perception deficiencies on the one hand and phonological and auditory deficiencies on the other, we constructed some additional variables indicating whether a specific skill is impaired or not. Because of the high intrasubject variability (even for scores measuring the same overall skill), averaging across these measures might underestimate the presence 


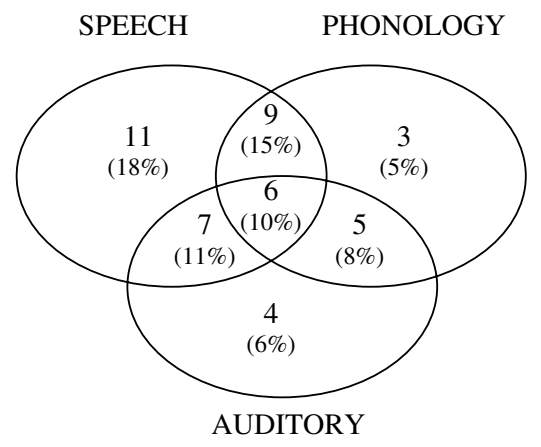

No deficits $=17(27 \%)$

Fig. 4. Distribution of auditory, speech perception and phonological deficits in the total sample of 62 preschool children. The number of impaired subjects is indicated both in absolute numbers and in percentages.

of a deficit in this skill. Instead, a skill was labeled as deficient if at least one of its measures scored beyond the 1.65 $S D$ cutoff criterion. As such, PHONOLOGY is a binary variable indicating whether a subject demonstrates a deficit in phonological awareness, rapid automatic naming or verbal short-term memory. Similarly, AUDITORY is a binary variable indicating whether a subject demonstrates a deficit in at least one of the auditory measures (viz. gap-in-noise, tone-in-noise and FM detection) and SPEECH is a binary variable indicating whether a subject shows deviant scores for SRT or for the slope parameter of the identification function. As mentioned above, some subjects were not able to pass the $70 \%$ pre-test or PSE $>1$ criterion of the identification task. Since we felt this failure might also be indicative for speech perception problems, these subjects were also labeled as deviant on the SPEECH variable.

Fig. 4 depicts a schematic overview of the number of subjects showing isolated versus (partially) overlapping deficits. Although a small group of subjects indeed presents consistent deficits across auditory, speech and phonological processing, the overview convincingly demonstrates that the overlap in deficits is certainly not as absolute as assumed. Indeed, contrary to the causal hypothesis, there are many subjects with severe deficits in only one of the skills while being unimpaired in the other ones, or subjects showing only partially overlapping deficits.

\section{Discussion}

In this study we tested speech perception and its relation with phonological and auditory processing in a group of five-year-old preschool children at family risk for dyslexia, compared to a group of well-matched control children. Additionally, children's speech perception abilities were also contrasted with those of adult subjects.

Comparing child and adult data, adults demonstrated better speech-in-noise perception, better categorical perception and better speech discriminability than children. These results are in line with the general literature regarding devel- opmental psychoacoustics (e.g. Wightman \& Allen, 1992), and have also been demonstrated for the specific speech perception tasks at hand (e.g. Elliot, 1979; Hazan \& Barrett, 2000; Johnson, 2000; Mills, 1975; Serniclaes et al., 2004).

Comparing both child groups with each other, the HR group presented a slight but significant deficit in speech-innoise perception, particularly in the most difficult listening condition. This result corroborates earlier data reporting a speech-in-noise perception deficit in subjects with dyslexia and/or language impairments (Bradlow et al., 2003; Brady et al., 1983; Chermak et al., 1989; Stollman et al., 1994). In particular, the observation that the HR children were relatively more affected by a decreasing signal-to-noise ratio is consistent with findings by Bradlow et al. (2003) and Stollman et al. (1994).

On the categorical perception task, neither of both child groups presented a clear categorical perception (as evidenced by the absence of a peak in the discrimination function and the generally shallow identification function). This suggests that five-year-olds in general have not yet developed a consistent phonemic categorization (see also Hazan $\&$ Barrett, 2000). Therefore, it is no surprise that both child groups did not differ on the identification task, and that the HR group only presented a marginally significant deficit on the discrimination task. On the other hand, it is not unthinkable that group differences might still emerge and become more evident as development continues. This would imply that identification and discrimination curves of normal reading children will evolve towards those of the adult group, whereas those of the dyslexic children will continue lagging behind.

Thus, so far, our group results provide rather limited evidence for a speech perception deficit in preschool children at family risk for dyslexia. This conclusion is confirmed by the results of the individual deviance analysis, indicating that although the HR group generally presented a higher proportion of deviant subjects, this difference was not significant. A possible explanation for the rather small difference between both child groups might be the fact that we did not study a well-defined clinical group but only an at-risk group.

Alternatively, it remains unclear to what extent the observed speech-in-noise perception deficit in the HR group reflects a genuine impairment in speech processing. Indeed, it has been demonstrated that much of the differences between adult and child auditory performance can be explained by a child's attention lapses (Oh, Wightman, \& Lutfi, 2001; Wightman, Callahan, Lutfi, Kistler, \& Oh, 2003). Correspondingly, given the high comorbidity between dyslexia and attention-related disorders (ADHD/ ADD, see e.g. Willcutt \& Pennington, 2000), it is plausible that the difference between the HR and LR group partly reflects attention differences.

In a previous paper describing the same preschool subjects (Boets et al., 2006), we already reported a considerable correlation between FM and TN detection and phonological awareness. Likewise, in the actual study we also observed a significant relation between speech perception 
on the one hand, and FM and TN detection and phonological awareness on the other. In contrast, the typically temporal GAP detection task was completely unrelated to any speech or phonological measure, suggesting that it is not specifically the temporal auditory aspect that determines the observed relations. Instead, it appears as if the common spectral or frequency sensitive characteristic of FM and TN detection determines the relation with speech perception and phonological ability.

However, the results of the individual deviance analysis demonstrate that the theoretical implications of these general relations between aspects of auditory processing, speech perception and phonological ability should not be overestimated. Indeed, contrary to the assumption of the auditory temporal processing deficit hypothesis, there was only a very small subgroup showing consistent deficiencies across all processing skills. The overall picture roughly indicated that about $30 \%$ of the subjects were unimpaired, $30 \%$ presented an isolated deficit, $30 \%$ presented a partially overlapping deficit in two skills, and a small subgroup of about $10 \%$ presented consistent deficiencies across the three processing skills. Regarding the proportion of HR subjects in these differently affected groups, an interesting pattern was revealed: whereas the HR subjects only represented $32 \%$ of the unaffected group, their proportion grew to $56 \%$ and $61 \%$ of the groups showing respectively one and two deficits, and they ultimately represented up to $100 \%$ of the group showing deficits across all the processing skills.

The observation that only a relatively small proportion of risk children presents speech perception problems, and in particular the observation that only a small group of subjects shows overlapping deficits in auditory processing, speech perception and phonology, calls for a revision or refinement of the theoretically postulated developmental pathway. Indeed, parallel to the growing evidence that only a small subgroup of dyslexics presents low-level auditory problems (Ramus et al., 2003; Rosen, 2003), there is also growing evidence that only a minority of dyslexics shows speech perception problems (Manis et al., 1997). For instance, Joanisse and colleagues (2000) studied three groups of dyslexics (a language impaired, a phonologically impaired and a globally reading delayed group) and demonstrated that speech perception problems only occur in dyslexic subjects showing additional expressive and/or receptive language problems. They also observed several dyslexics with phonological impairments whose speech perception was normal, confirming that phonological impairments are not necessarily secondary to a speech perception deficit. Accordingly, these authors argued that although the language impaired and phonologically impaired dyslexic groups exhibited similar deficits in phonology and reading, their causes may differ, with one involving a speech perception deficit and the other involving higher-order differences in phonological representation or processing. The third globally reading delayed group of their sample did not show any deficits in speech perception or phonology at all, suggesting still another cause for their reading deficit. Therefore, together with these authors we would contend that in order to fully understand the deviant developmental pathway of dyslexia, it will be necessary to take into account the considerable heterogeneity of this group and to recognize that subjects presenting similar patterns of reading problems must not necessarily have the same underlying deficits. In this way it is plausible that the hypothesized causal path (from auditory processing to speech perception to phonology to reading) might be true for a specific subgroup of dyslexics, but it probably is not representative for the whole population of reading impaired subjects.

\section{Acknowledgments}

This research was financed by the Fund for Scientific Research-Flanders (Belgium), Grant G.0216.02. We thank the children and school staff who were involved in this study. Further, we thank Kathelijne Jordens for assistance with data collection, Lieve Helsen for assistance with E-prime programming, Willy Leung for putting a lot of creative effort in creating the animation movies and we especially show gratitude to the speech research team of the Dutch Dyslexia Research Program (NWO, 1996) for making their speech stimuli available to us. Finally, we thank F. Wightman and two anonymous reviewers for helpful comments on previous drafts of this paper.

\section{Appendix A}

Individual $Z$-scores relative to the mean of the 'restricted' LR group for speech perception, phonology and auditory processing. Scores deviating more than 1.65 SD's (in the deficient direction) are depicted in bold

\begin{tabular}{|c|c|c|c|c|c|c|c|c|c|c|c|c|}
\hline $\mathrm{Nr}$ & Group & SRT & $\begin{array}{l}\text { Slope phoneme } \\
\text { boundary }^{\mathrm{a}}\end{array}$ & $\mathrm{TN}$ & $\mathrm{GAP}^{\mathrm{a}}$ & $\mathrm{FM}^{\mathrm{a}}$ & $\begin{array}{l}\text { Phon } \\
\text { awareness }\end{array}$ & RAN & $\begin{array}{l}\text { Verbal } \\
\text { STM }\end{array}$ & Auditory & Phonology & Speech \\
\hline 1 & HR & -0.41 & -1.15 & -0.11 & -0.16 & 1.07 & 0.46 & 0.27 & 0.56 & & & \\
\hline 3 & HR & 1.31 & & 0.68 & -0.90 & -1.46 & -1.64 & 0.23 & 0.32 & & & Deviant \\
\hline 5 & HR & 0.73 & -2.53 & 0.42 & -0.92 & 2.08 & -0.83 & 0.85 & -0.36 & Deviant & & Deviant \\
\hline 7 & HR & -0.47 & -2.26 & 0.69 & -0.68 & 0.19 & -1.61 & -0.63 & 1.15 & & & Deviant \\
\hline 9 & HR & 0.00 & 0.41 & -1.87 & -1.16 & -1.34 & 0.89 & -0.56 & -1.03 & & & \\
\hline 11 & HR & 1.99 & & 0.85 & 1.33 & 0.73 & -0.23 & -0.51 & -0.02 & & & Deviant \\
\hline 13 & HR & 1.55 & -0.40 & 0.34 & -0.33 & -0.32 & 0.20 & 0.40 & -0.10 & & & \\
\hline
\end{tabular}


Appendix A (continued)

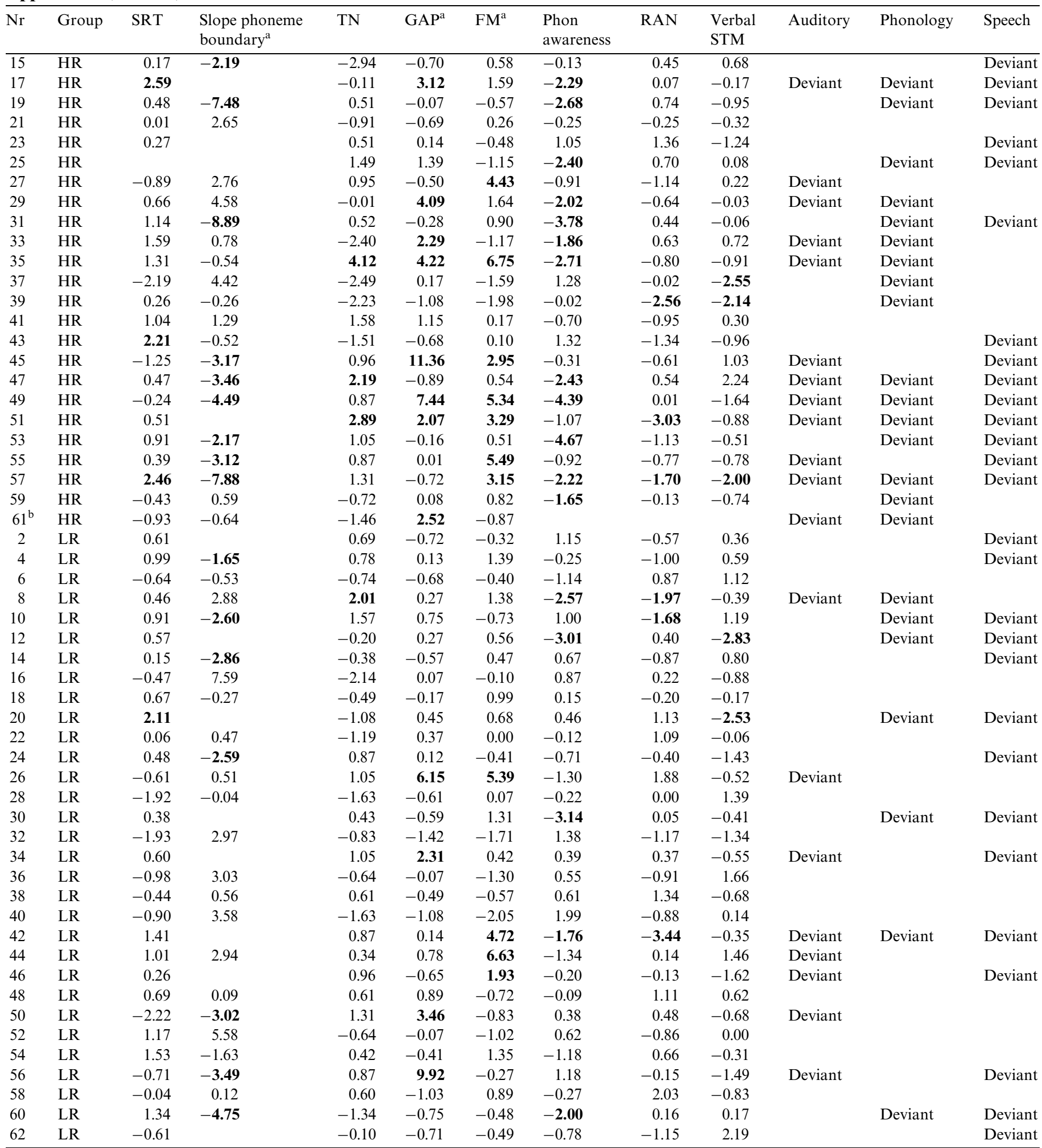

Note. For the phoneme boundary slope, phonological awareness, RAN and Verbal STM a higher $Z$-score indicates better processing; in contrast, for all other measures a higher $Z$-score indicates reduced sensory sensitivity.

${ }^{\text {a }}$ Scores were $\log _{10}$-transformed prior to analysis.

${ }^{\mathrm{b}}$ Because of missing scores on two phonological tests, no phonological factors could be calculated for subject 61 . Notwithstanding, based on the scores of the administered tests, it was determined that this subject showed a deficit in phonological awareness. 


\section{References}

Bailey, P., Griffiths, Y., Hill, N., Brent, S., \& Snowling, M. (2002). Speech intelligibility in continuous and intermittent noise for dyslexic and normally reading listeners. Poster Presented at the ISCA Workshop on Temporal Integration in the Perception of Speech (TIPS), Aix-EnProvence, 8th-10th April.

Blomert, L., \& Mitterer, H. (2004). The fragile nature of the speech-perception deficit in dyslexia: natural versus synthetic speech. Brain and Language, 89, 21-26.

Boets, B., Wouters, J., van Wieringen, A., \& Ghesquière, P. (2006). Auditory temporal information processing in preschool children at family risk for dyslexia: relations with phonological abilities and developing literacy skills. Brain and Language, 97, 64-79.

Bowers, P. G., \& Swanson, L. B. (1991). Naming speed deficits in reading disability: multiple measures of a singular process. Journal of Experimental Child Psychology, 51, 195-219.

Bradlow, A. R., Kraus, N., \& Hayes, E. (2003). Speaking clearly for children with learning disabilities: sentence perception in noise. Journal of Speech, Language and Hearing Research, 46, 80-97.

Brady, S., Shankweiler, D., \& Mann, V. (1983). Speech perception and memory coding in relation to reading ability. Journal of Experimental Child Psychology, 35(2), 345-367.

Brandt, J., \& Rosen, J. J. (1980). Auditory phonemic perception in dyslexia: categorical identification and discrimination of stop consonants. Brain and Language, 9, 324-337.

Breier, J. I., Fletcher, J. M., Denton, C., \& Gray, L. C. (2004). Categorical perception of speech stimuli in children at risk for reading difficulty. Journal of Experimental Child Psychology, 88, 152-170.

Catts, H. W. (1989). Phonological processing deficits and reading disabilities. In A. G. Kahmi \& H. W. Catts (Eds.), Reading disabilities: A developmental language perspective (pp. 101-132). Boston, MA: Littel Brown.

Chermak, G. D., Vonhof, M. R., \& Bendel, R. B. (1989). Word identification performance in the presence of competing speech and noise in learning disabled adults. Ear and Hearing, 10(2), 90-93.

Cunningham, J., Nicol, T., Zecker, S. G., Bradlow, A., \& Kraus, N. (2001). Neurobiologic responses to speech in noise in children with learning problems: deficits and strategies for improvement. Clinical Neurophysiolgy, 112(5), 758-767.

Denenberg, V. H. (1999). A critique of Mody, Studdert-Kennedy, and Brady's "Speech perception deficits in poor readers: auditory processing or phonological coding?". Journal of Learning Disabilities, 32(5), 379-383.

Elliot, L. L. (1979). Performance of children aged 9 to 17 on a test of speech intelligiblity in noise using sentence material with controlled word predictability. Journal of the Acoustical Society of America, 66, 651-653.

Farmer, M. E., \& Klein, R. M. (1995). The evidence for a temporal processing deficit linked to dyslexia: A review. Psychonomic Bulletin and Review, 2(4), 460-493.

Gerrits, E. (2003). Speech perception of young children at risk for dyslexia and children with specific language impairment. Proceedings of the 15th International Conference on Phonetic Sciences (pp. 2357-2360). Barcelona, Spain, 3 - 9 Augustus 2003.

Gilger, J. W., Pennington, B. F., \& DeFries, J. C. (1991). Risk for reading disability as a function of parental history in three family studies. Reading and Writing, 3(3-4), 205-217.

Godfrey, J. J., Syrdal-Lasky, A. K., Millay, K. K., \& Knox, C. M. (1981). Performance of dyslexic children on speech perception tests. Journal of Experimental Child Psychology, 32, 401-424.

Hazan, V., \& Barrett, S. (2000). The development of phonemic categorization in children aged 6-12. Journal of Phonetics, 28, 377-396.

Joanisse, M. F., Manis, F. R., Keating, P., \& Seidenberg, M. S. (2000). Language deficits in dyslexic children: speech perception, phonology and morphology. Journal of Experimental Child Psychology, 77, 30-60.

Johnson, C. E. (2000). Children's phoneme identification in reverberation and noise. Journal of Speech, Language and Hearing Research, 43, 144-157.
Laneau, J., Boets, B., Moonen, M., van Wieringen, A., \& Wouters, J. (2005). A flexible auditory research platform using acoustic or electric stimuli for adults and young children. Journal of Neuroscience Methods, 142(1), 131-136.

Liberman, A. M., Harris, K. S., Hoffman, H. S., \& Griffith, B. C. (1957). The discrimination of speech sounds within and across phoneme boundaries. Journal of Experimental Psychology, 54, 358-368.

Lieberman, P., Meskill, R. H., Chatillon, M., \& Schupack, H. (1985). Phonetic speech perception deficits in dyslexia. Journal of Speech and Hearing Research, 28, 480-486.

Littell, R. C., Stroup, W. W., \& Freund, R. J. (2002). SAS for linear models. Cary: SAS Institute.

Lyytinen, H., Ahonen, T., Eklund, K., Guttorm, T. K., Laakso, M. L., Leinonen, S., et al. (2001). Developmental pathways of children with and without familial risk for dyslexia during the first years of life. Developmental Neuropsychology, 20(2), 535-554.

Maassen, B., Groenen, P., Crul, T., Assman-Hulsmans, C., \& Gabreëls, F. (2001). Identification and discrimination of voicing and place-of-articulation in developmental dyslexia. Clinical Linguistics and Phonetics, 15(4), 319-339.

Manis, F. R., Custodio, R., \& Szeszulski, P. A. (1993). Development of phonological and orthographic skills: a 2-year longitudinal study of dyslexic children. Journal of Experimental Child Psychology, 56, 64-86.

Manis, F. R., McBride-Chang, C., Seidenberg, M. S., Keating, P., Doi, L. M., Munson, B., et al. (1997). Are speech perception deficits associated with developmental dyslexia? Journal of Experimental Child Psychology, 66, 211-235.

McArthur, G. M., \& Bishop, D. V. (2001). Auditory perceptual processing in people with reading and oral language impairments: current issues and recommendations. Dyslexia, 7(3), 150-170.

McBride-Chang, C. (1995). Phonological processing, speech perception and reading disability: an integrative review. Educational Psychologists, 30(3), 109-121.

Menell, P., McAnally, K. I., \& Stein, J. F. (1999). Psychophysical sensitivity and physiological response to amplitude modulation in adult dyslexic listeners. Journal of Speech, Language and Hearing Research, 42(4), 797-803.

Mills, J. H. (1975). Noise and children: a review of literature. Journal of the Acoustical Society of America, 58, 767-779.

Mody, M., Studdert-Kennedy, M., \& Brady, S. (1997). Speech perception deficits in poor readers: auditory processing or phonological coding? Journal of Experimental Child Psychology, 64(2), 199-231.

Nittrouer, S. (1999). Do temporal processing deficits cause phonological problems? Journal of Speech, Language and Hearing Research, 42, 925-942.

Oh, E. L., Wightman, F. L., \& Lutfi, R. A. (2001). Children's detection of pure-tone signals with random multitone maskers. Journal of the Acoustical Society of America, 109, 2888-2895.

Pennington, B. F., Van Orden, G. C., Smith, S. D., Green, P. A., \& Haith, M. M. (1990). Phonological processing skills and deficits in adult dyslexics. Child Development, 61, 1753-1778.

Rack, J. P. (1994). Dyslexia: The phonological deficit hypothesis. In A. Fawcett \& R. Nicolson (Eds.), Dyslexia in children: Multidisciplinary perspectives. London: Harvester Wheatsheaf.

Ramus, F., Rosen, S., Dakin, S. C., Day, B. L., Castellote, J. M., White, S., et al. (2003). Theories of developmental dyslexia: insights from a multiple case study of dyslexic adults. Brain, 126(4), 841-865.

Raven, J. C., Court, J. H., \& Raven, J. (1984). Manual for Raven's Progressive Matrices and Vocabulary Scales. London: Lewis.

Reed, M. A. (1989). Speech perception and the discrimination of brief auditory cues in reading disabled children. Journal of Experimental Child Psychology, 48(2), 270-292.

Richardson, U., Leppänen, P. H. T., Leiwo, M., \& Lyytinen, H. (2003). Speech perception of infants with high familial risk for dyslexia differ at the age of 6 months. Developmental Neuropsychology, 23(3), 385-397.

Rosen, S. (2003). Auditory processing in dyslexia and specific language impairment: is there a deficit? What is its nature? Does it explain anything? Journal of Phonetics, 31, 509-527. 
Rosen, S., \& Manganari, E. (2001). Is there a relationship between speech and nonspeech auditory processing in children with dyslexia? Journal of Speech, Language and Hearing Research, 44, 720-736.

Scarborough, H. S. (1998). Early identification of children at risk for reading disabilities: phonological awareness and some other promising predictors. In P. J. Accardo, A. J. Capute, \& B. K. Shapiro (Eds.), Specific reading disability: A view of the spectrum (pp. 75-119). Timonium, MD: York Press.

Schneider, W., Eschman, A., \& Zuccolotto, A. (2002). E-Prime User's Guide. Pittsburgh: Psychology Software Tools Inc..

Serniclaes, W., Sprenger-Charolles, L., Carré, R., \& Demonet, J. (2001). Perceptual discrimination of speech sounds in developmental dyslexia. Journal of Speech, Language and Hearing Research, 44, 384-399.

Serniclaes, W., Van Heghe, S., Mousty, P., Carré, R., \& Sprenger-Charolles, L. (2004). Allophonic mode of speech perception in dyslexia. Journal of Experimental Child Psychology, 87, 336-361.

Snowling, M. J. (2000). Dyslexia (2nd ed.). Malden, MA: Blackwell Publishers.

Snowling, M., Goulandris, N., Bowlby, M., \& Howell, P. (1986). Segmentation and speech perception in relation to reading skill: a developmental analysis. Journal of Experimental Child Psychology, 41(3), 489-507.

Steffens, M. L., Eilers, R. E., Gross-Glenn, K., \& Jallad, B. (1992). Speech perception in adult subjects with familial dyslexia. Journal of Speech and Hearing Research, 35, 192-200.

Stollman, M., Kapteyn, T., \& Sleeswijk, B. (1994). Effect of time-scale modification of speech on the speech recognition threshold in noise for hearing-impaired and language-impaired children. Scandinavian Audiology, 23, 39-46.

Talcott, J. B., \& Witton, C. (2002). A sensory linguistic approach to the development of normal and impaired reading skills. In E. Witruk, A. Friederici, \& T. Lachmann (Eds.), Neuropsychology and cognition series. Basic functions of language and language disorders. Dordrecht, Netherlands: Kluwer Academic Publishers.

Talcott, J. B., Witton, C., McClean, M., Hansen, P. C., Rees, A., Green, G., et al. (1999). Can sensitivity to auditory frequency modulation predict children's phonological and reading skills? Neuroreport, 10(10), 20452050.

Talcott, J. B., Witton, C., McLean, M. F., Hansen, P. C., Rees, A., Green, G. G., et al. (2000). Dynamic sensory sensitivity and children's word decoding skills. Proceedings of the National Academy of Science of the United States of America, 97(6), 2952-2957.

Talcott, J. B., Witton, C., Hebb, G. S., Stoodley, C. J., Westwood, E. A., France, S. J., et al. (2002). On the relationship between dynamic visual and auditory processing and literacy skills; results from a large primary-school study. Dyslexia, 8(4), 204-225.

Tallal, P. (1980). Auditory temporal perception, phonics, and reading disabilities in children. Brain and Language, 9(2), 182-198.

Tallal, P. (1984). Temporal or phonetic processing deficit in dyslexia? That's the question. Applied Psycholinguistics, 5, 167-169.

van Beinum, F. J., Schwippert, C. E., Been, P. H., van Leeuwen, T. H., \& Kuijpers, C. T. L. (2005). Development and application of a /bAk/-/
$\mathrm{dAk} /$ continuum for testing auditory perception within the Dutch longitudinal study. Speech Communication, 47, 124-142.

Van Ingelghem, M., Boets, B., van Wieringen, A., Onghena, P., Ghesquière, P., \& Wouters, J. (2005). An auditory temporal processing deficit in children with dyslexia? In P. Ghesquière \& A. J. J. M. Ruijssenaars (Eds.), Learning disabilities: A challenge to teaching and instruction. Series: Studia Paedagogica 40 (pp. 47-63). Leuven: University Press.

van Wieringen, A., \& Wouters, J. (2005). Normalization and feasibility of speech understanding tests for Dutch speaking toddlers. Speech Communication, 47, 169-181.

Wagner, R. K., \& Torgesen, J. K. (1987). The nature of phonological processing and its causal role in the acquisition of reading skills. Psychological Bulletin, 101(2), 192-212.

Warrier, C. M., Johnson, K. L., Hayes, E. A., Nicol, T., \& Kraus, N. (2004). Learning impaired children exhibit timing deficits and training-related improvements in auditory cortical responses to speech in noise. Experimental Brain Research, 157, 431-441.

Watson, B. U., \& Miller, T. K. (1993). Auditory perception, phonological processing and reading ability/disability. Journal of Speech and Hearing Research, 36(4), 850-863.

Werker, J. F., \& Tees, R. C. (1987). Speech perception in severely disabled and average reading children. Canadian Journal of Experimental Psychology, 41, 48-61.

Wible, B., Nicol, T., \& Kraus, N. (2002). Abnormal neural encoding of repeated speech stimuli in noise in children with learning problems. Clinical Neurophysiology, 113(4), 485-494.

Wightman, F., \& Allen, P. (1992). Individual differences in auditory capability among preschool children. In L. A. Werner \& E. W. Rubel (Eds.), Developmental psychoacoustics (pp. 113-134). Washington DC: American Psychological Association.

Wightman, F. L., Callahan, M. R., Lutfi, R. A., Kistler, D. J., \& Oh, E. (2003). Children's detection of pure-tone signals: informational masking with contralateral maskers. Journal of the Acoustical Society of America, 113(6), 3297-3305.

Willcutt, E. G., \& Pennington, B. F. (2000). Comorbidity of reading disability and attention deficit/hyperactivity disorder: differences by gender and subtype. Journal of Learning Disabilities, 33, 179-191.

Witton, C., Talcott, J. B., Hansen, P. C., Richardson, A. J., Griffiths, T. D., Rees, A., et al. (1998). Sensitivity to dynamic auditory and visual stimuli predicts nonword reading ability in both dyslexic and normal readers. Current Biology, 8(14), 791-797.

Wolf, M. (1986). Rapid alternating stimulus naming in the developmental dyslexias. Brain and Language, 27, 360-379.

Wood, C. C. (1976). Discriminability, response bias and phoneme categories in discrimination of voice onset time. Journal of the Acoustic Society of America, 60(6), 1381-1389.

Wouters, J., Damman, W., \& Bosman, A. J. (1994). Vlaamse opnamen van woordenlijsten voor spraakaudiometrie. CD NKO - K.U.Leuven/ U.Z.Leuven.

Wright, B. A., Lombardino, L. J., King, W. M., Puranik, C. S., Leonard, C. M., \& Merzenich, M. M. (1997). Deficits in auditory temporal and spectral resolution in language-impaired children. Nature, 387, 176-178. 